\title{
Band structure in Yang-Mills theories
}

\author{
Constantin Bachas ${ }^{a}$ and Theodore Tomaras ${ }^{b}$ \\ ${ }^{a}$ Laboratoire de Physique Théorique de l'École Normale Supérieure, \\ PSL Research University, CNRS, Sorbonne Universités, UPMC Univ. Paris 06, \\ 24 rue Lhomond, 75231 Paris Cedex 05, France \\ ${ }^{b}$ Department of Physics and Institute for Theoretical and Computational Physics, \\ University of Crete, 70013 Heraklion, Greece \\ E-mail: bachas@lpt.ens.fr, tomaras@physics.uoc.gr
}

ABSTRACT: We show how Yang-Mills theory on $\mathrm{S}^{3} \times \mathbb{R}$ can exhibit a spectrum with continuous bands if coupled either to a topological 3-form gauge field, or to a dynamical axion with heavy Peccei-Quinn scale. The basic mechanism consists in associating winding histories to a bosonic zero mode whose role is to convert a circle in configuration space into a helix. The zero mode is, respectively, the holonomy of the 3 -form field or the axion momentum. In these models different $\theta$ sectors coexist and are only mixed by (non-local) volume operators. Our analysis sheds light on, and extends Seiberg's proposal for modifying the topological sums in quantum field theories. It refutes a recent claim that $B+L$ violation at LHC is unsuppressed.

KEYwords: Nonperturbative Effects, Solitons Monopoles and Instantons, Topological Field Theories

ARXIV EPRINT: 1603.08749 


\section{Contents}

1 Introduction 1

2 Circle versus helix 2

3 Chern-Simons number as helix angle $\quad 5$

4 Dynamical axion $\quad 8$

5 Concluding remarks 10

$\begin{array}{ll}\text { A An action for the Chern-Simons number } & 11\end{array}$

\section{Introduction}

While revisiting recently the question of electroweak $B+L$ violation [1] in collider experiments (for reviews and more references see [2-7]) Tye and Wong made a bold proposal [8, 9]. They argued that the relevant degree of freedom in the space-time interaction region for such processes is the Chern-Simons number of the electroweak gauge fields, whose effective quantum mechanics exhibits band structure similar to that of an electron in a solid. They further argued that the width of these bands is proportional to the amplitude of the instanton-induced tunneling transitions which grows with available energy, and went on to conclude that $B+L$ violation may be unsuppressed at LHC.

This last conclusion misses in our opinion the key aspect of the problem: the difficulty of streamlining the collision energy into coherent excitations of one or few quantum degrees of freedom. In weakly-coupled theories such streamlining is expected to be exponentially small, independently of any other features such as the existence of many vacua. Thus, even though the inclusive cross-section for tunneling processes does grow with collision energy $[10,11]$ the growth is believed to stop much before one hits the unitarity bound. This can be shown rigorously in an analog $\lambda \phi^{4}$ quantum mechanical model [12-14].

Quite independently of LHC physics, the claim that Yang-Mills theory can exhibit band structure is by itself surprising. Energy bands arise when a particle moves in a noncompact dimension with periodic potential $V(q)=V(q+n), n \in \mathbb{Z}$. A band is spanned by the quasi-momentum (or lattice momentum) which takes values in a Brillouin zone. In the Yang-Mills case the coordinate $q$ is the Chern-Simons number of the gauge field, and the shifts $q \rightarrow q+n$ are implemented by large gauge transformations. Since these are gauge and not global symmetries, $q$ is identified with $q+1$ and the wavefunction must be a periodic function of $q$. This fixes the quasi-momentum, so there are no bands.

The purpose of this short letter will be to discuss how to evade this conclusion. The basic idea is to associate tunneling transitions to a bosonic zero mode which provides a 
'tag' that distinguishes the winding vacua. This device effectively converts large gauge transformations into global symmetries or, more figuratively, turns the $q$-circle into a (noncompact) helix for which the spectrum exhibits (continuous) bands. The question then is whether this strategy can be implemented within local quantum field theory. We will discuss two different ways of doing this on a compact space manifold.

The first, proposed by Seiberg [15], involves coupling Yang-Mills theory to a topological 3 -form gauge field $B$. What tags the winding vacua is the holonomy $b=\int_{M_{3}} B$ of $B$ around the space manifold $M_{3}$. Another, less contrived but only approximate way involves coupling Yang-Mills to a dynamical axion, $a$, with very large decay constant $f_{a}$. The linear coordinate of the helix is in this case the axion momentum or, equivalently, the integrated Poincaré dual 3-form $\int_{M_{3}}{ }^{*} d a$. As we will see, the axion model (which arises naturally from string theory) reduces to the topological model in the limit $f_{a} \rightarrow \infty$.

The bands in the above extensions of gauge theory are filled by coexisting $\theta$ sectors, which are not mixed by local operators but do talk via charged-membrane interface probes (3-dimensional analogs of Wilson lines). Even under the optimistic assumptions (1) that we may replace the 3 -sphere in our analysis by an interaction region of size $m_{W}^{-1}$, and (2) that the probe membranes become dynamical, these latter would be too heavy to play any role in collider physics. Thus, independently of any other objections, the band structure that we exhibit here is not relevant for $B+L$ violation at LHC. Nevertheless our results provide new insights on the topological sector of non-abelian gauge theories, and the 'helix mechanism' is so simple and general that it may find applications in other contexts.

The paper is organized as follows. In section 2 we describe the basic idea with the example of a charged particle moving on a helix or on a circle, in a gravitational potential. Because $2 \pi$ rotations are global (respectively gauge) symmetries, the particle's spectrum does (does not) have energy bands. In section 3 we explain how Seiberg's proposal to couple Yang-Mills to a topological 3-form gauge field implements the helix mechanism in local quantum field theory. Section 4 presents a different realization which involves a dynamical axion with high Peccei-Quinn scale $f_{a}$. We show that in this theory approximate $\theta$ sectors coexist, and that in the limit $f_{a} \rightarrow \infty$ one recovers the non-compact version of Seiberg's model. Finally, section 5 contains some concluding remarks, in particular on electroweak $B+L$ violation - the issue that prompted this investigation.

A quantum-mechanical action for the Chern-Simons variable, similar to the one proposed by Tye and Wong [8], can be derived by putting the SU(2) theory on the 3-sphere and then projecting onto the $\mathrm{SO}(4)$-invariant sector. This is an amusing exercise, but since the details of this action play no role we relegate it to the appendix.

\section{Circle versus helix}

One reads in many textbooks that Yang-Mills theory has winding vacua $|n\rangle$, that the true vacuum is a linear superposition $|\theta\rangle=\sum_{n} e^{i n \theta}|n\rangle$, and that different $|\theta\rangle$ belong to distinct superselection sectors. Though usually a matter of semantics, these statements hide a subtle aspect of the problem and can be misleading. We start by explaining why in a 

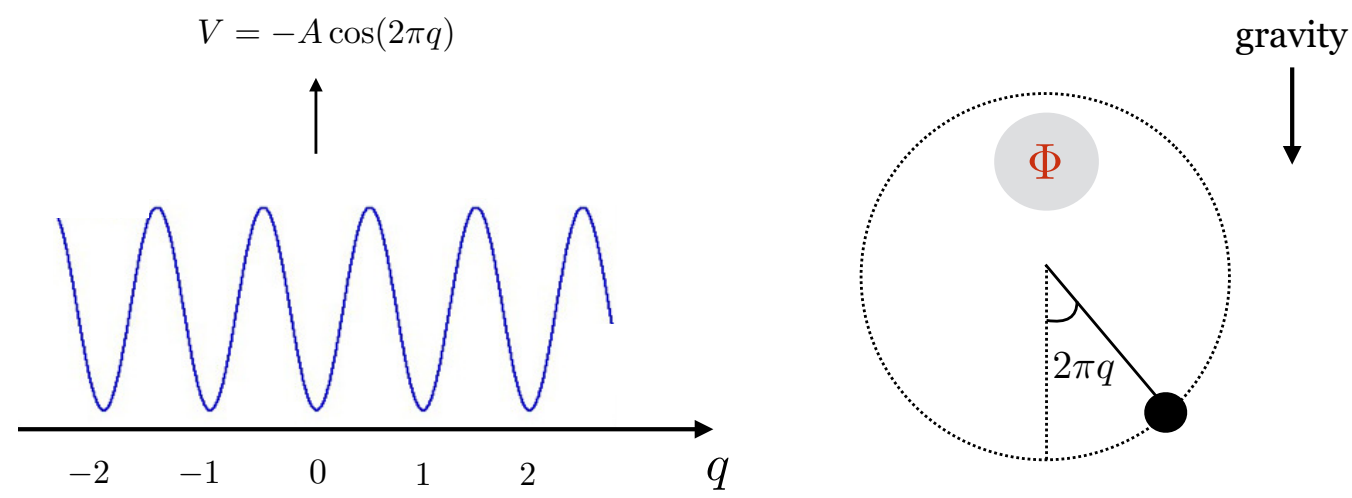

Figure 1. A particle moving on the real line in the potential $V=-A \cos (2 \pi q)$ has the same action as a pendulum of unit radius with $A=M \times$ (Earth's gravity). In the pendulum the discrete symmetry under $2 \pi$ rotations is gauged and $V$ has a unique minimum, whereas on the real line the symmetry is global and the minima are distinct. The magnetic flux $\Phi$ shown in the figure induces a theta term with $\theta=e \Phi$, where $e$ is the particle's charge.

simple model of quantum mechanics. This is standard material (see e.g. [17]) and readers may want to go quickly to the next section.

The model is that of a particle moving in a periodic potential $V(q)=V(q+n), n \in \mathbb{Z}$ with action

$$
S=\int d t \mathcal{L}=\int d t\left[\frac{M}{2} \dot{q}^{2}-V(q)-\theta \dot{q}\right]
$$

We have added to $\mathcal{L}$ a topological term that plays the role of the $\theta$ term in Yang-Mills theory, as will be clear in the following section. To fully specify the model we must still state whether the symmetry under discrete translations, $q \rightarrow q+n$, is global or gauged. ${ }^{1}$ In the former case the particle lives on the real line and the potential has an infinite number of local minima labelled by $n \in \mathbb{Z}$, while in the latter it lives on a circle and $V$ has a unique minimum which we may choose to be at $q=0$.

A physical realization of the circle model is a quantum pendulum moving in the Earth's gravitational field. The topological $\theta$ term arises if the pendulum carries electric charge and encircles a magnetic flux. To turn the gauge symmetry of $2 \pi$ rotations into a global symmetry one can employ a simple trick: convert the circle into a helix by forcing the particle to move in an extra (horizontal) dimension $z$, so that $z=\xi q$. The minima of the potential are now distinguished by their position in the extra dimension, and $q$ is effectively decompactified. The $\theta$ term still corresponds to a flux tube threading the helix. These facts are illustrated in the figure 1 above.

The physics of these problems is familiar. In the non-compact case the energy eigenstates are organized in continuous bands, while in the compact case one keeps a single Bloch wave in every band and the spectrum is discrete. At the risk of being pedantic, let us recall

\footnotetext{
${ }^{1}$ More generally it is possible to only gauge a subgroup $q \rightarrow q+N n$ of the symmetry group which amounts to putting the particle on a circle of radius $N / 2 \pi$.
} 


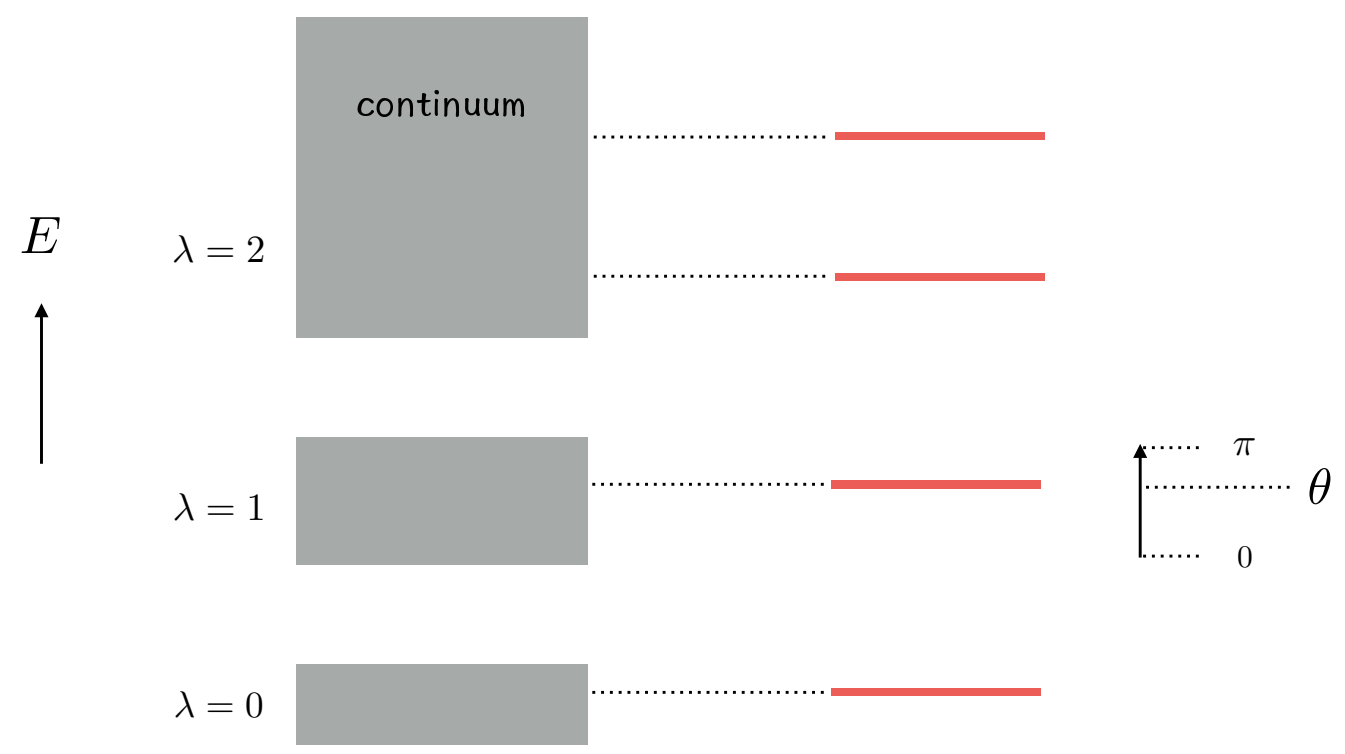

\section{Lattice}

\section{Pendulum}

Figure 2. On the left the typical band structure for a particle moving in a periodic potential on the real line (assuming $k \rightarrow-k$ invariance). The width of the bands grows with the tunneling amplitude between neighbouring wells and bands eventually merge in a continuum. Compactifying the line to a circle projects onto states with effective quasi-momentum $\theta$ (the red spectrum on the right).

how this works in more detail. From (2.1) one finds the momentum and Hamiltonian

$$
p \equiv \frac{\partial \mathcal{L}}{\partial \dot{q}}=M \dot{q}-\theta, \quad \mathcal{H}(\theta) \equiv p \dot{q}-\mathcal{L}=\frac{1}{2 M}(p+\theta)^{2}+V(q)
$$

The $\theta$-dependence looks, at first sight, trivial since it is removed by a unitary transformation

$$
p+\theta=e^{-i \theta q} p e^{i \theta q} \Longrightarrow \mathcal{H}(\theta)=e^{-i \theta q} \mathcal{H}(0) e^{i \theta q} .
$$

Indeed, $e^{i \theta q}$ is the Bohm-Aharonov phase for a particle moving around a magnetic flux tube as in figure 1. However, while $e^{i \theta q}$ is a legitimate operator in the helix model, it is not for the pendulum because it is multi-valued on the circle (unless $\theta \in 2 \pi \mathbb{Z}$ ). Thus $\theta$ is a relevant parameter in the compact case, whereas it can be absorbed by a redefinition of momentum in the non-compact case.

Either way, the generator of discrete translations, $P:=e^{i p}$, commutes with $\mathcal{H}$ and can be diagonalized. Its eigenvalues are phases $e^{i k}$, where $k \in[-\pi, \pi]$ is the latticemomentum or quasi-momentum. Energy eigenstates of the helix model are labelled by $k$ and by the index $\lambda=0,1, \cdots$ that designates an 'electron' band. A generic spectrum is illustrated in figure 2 . It has narrow 'tight-binding' bands whose width grows with $\lambda$, until they eventually merge in a continuum. If $E_{k, \lambda}(\theta)$ and $\psi_{k, \lambda}(q, \theta)$ are the eigenvalues and eigenfunctions of $\mathcal{H}(\theta)$, the unitary equivalence (2.3) implies

$$
\psi_{k, \lambda}(q, \theta)=e^{-i \theta q} \psi_{k+\theta, \lambda}(q, 0) \quad \text { and } \quad E_{k, \lambda}(\theta)=E_{k+\theta, \lambda}(0) .
$$


Thus the only effect of $\theta$ in the non-compact case is to reshuffle the eigenvalues in each energy band by a universal shift of $k$ - a trivial effect as stated above.

The situation is different in the compact case where $P$ is a gauge transformation, so it must leave invariant all physical states. We must now project to $k=0$, i.e. keep only the periodic wavefunctions $\psi_{0, \lambda}(q, \theta)$ whose energy is $E_{0, \lambda}(\theta)=E_{\theta, \lambda}(0)$. This keeps one state in each energy band as illustrated in the figure. If we use as reference the non-compact theory with $\theta=0$, then gauging the $\mathbb{Z}$ symmetry has the effect of projecting onto the sector with quasi-momentum equal to $\theta$.

More generally, one may gauge a subgroup $\{q \rightarrow q+n N \mid n \in \mathbb{Z}\}$ of the global symmetry and project onto $N$ states with equally-spaced quasi-momenta in each energy band. In the language of the helix, this amounts to taking $z$ periodic $(z \sim z+1)$ and $\xi=1 / N$ so that the helix has a total of $N$ turns. For $N \gg 1$ the bands are filled very densely. Alternatively, we may perturb the infinite-helix model by a shallow potential $\Delta V \sim \epsilon z^{2}$ with $\epsilon \ll 1$. Its effect is to cut off effectively the number of helix turns to $N \sim 1 / \sqrt{\epsilon}$, so the energy spectrum is to a good approximation the same as for a long periodic helix. We will see that both models are realized in gauge theory.

One final comment concerns the width of the bands, which measures the $\theta$-dependence of the spectrum in the circle model. For the low-lying bands this is proportional to the amplitude of tunneling between nearby potential wells which is exponentially small. At higher energies the particle gets delocalized and the bandwidth grows. A frequently used quantity is the topological susceptibility $\chi$, which is the second derivative of the groundstate energy at $\theta=0$. This vanishes when tunneling is suppressed, and it is exponentially small for a potential barrier that is hard to penetrate, $\chi \sim \exp \left(-S_{0}\right)$ where $S_{0}$ is the instanton action. For a freely-moving particle $\chi=1 / M .^{2}$

\section{Chern-Simons number as helix angle}

The periodic variable in Yang-Mills theory is the Chern-Simons number of the gauge field. In temporal gauge, appropriate for Hamiltonian quantization, this is defined by the wellknown expression

$$
n_{\mathrm{CS}}=-\frac{1}{8 \pi^{2}} \int d^{3} x \epsilon^{i j k} \operatorname{tr}\left(A_{i} \partial_{j} A_{k}+\frac{2}{3} A_{i} A_{j} A_{k}\right):=\int_{\mathrm{S}^{3}} \mathcal{C}(A),
$$

where $\mathcal{C}(A)$ is the shorthand symbol for the normalized Chern-Simons 3-form and we have taken space to be the 3 -sphere $S^{3}$. The Chern-Simons number is invariant under gauge transformations that are homotopic to the identity, but it transforms under large ones by integer shifts, $n_{\mathrm{CS}} \rightarrow n_{\mathrm{CS}}+n$, where $n$ is the winding number of the gauge transformation. Since all of the gauge transformations must act trivially on physical states, $n_{\mathrm{CS}}$ lives on the circle and is hence compact.

\footnotetext{
${ }^{2}$ The ground state energy of a free particle is $\theta^{2} / 2 M$. It has a cusp at $\theta= \pm \pi$, a fact that can be attributed more generally to the existence of many degenerate states [18]. We thank Cesar Gomez for this remark.
} 
Let us now assume, following Tye and Wong [8, 9], that it makes sense to write down an effective action for the variable $n_{\mathrm{CS}}$. We will comment on the validity of this assumption in the end. For now we only require two topological features of the action: that it is invariant under integer shifts, and that its potential be bounded. The maximum of the potential in the electroweak theory is the sphaleron's mass [19]. In the pure gauge theory considered here the infrared cutoff is the inverse sphere radius that we set equal to one, so the potential barrier should be of order $\sim 1 / g^{2}$.

For the sake of concreteness, we may write down an action by truncating the theory on $\mathrm{S}^{3}$ to the $\mathrm{SO}(4)$-invariant sector. ${ }^{3}$ This reduces configuration space to a single variable, which can be chosen conveniently to be $n_{\mathrm{CS}}$. The action is then fixed by requiring that the standard Belavin et al. instanton [20] be a solution of the reduced model in imaginary time [21]. The exercise is worked out in appendix A. The result is more easily expressed after a non-linear redefinition of the Chern-Simons variable,

$$
n_{\mathrm{CS}}(q)=q^{2}(3-2 q),
$$

where $q$ must be restricted to the range $[0,1]$ and

$$
S(q, \dot{q})=\frac{12 \pi^{2}}{g^{2}} \int d t\left[\dot{q}^{2}-4 q^{2}(q-1)^{2}\right]-\theta \int d t \dot{n}_{\mathrm{CS}}(q) .
$$

Note that as $n_{\mathrm{CS}}$ varies from 0 to 1 , the variable $q$ covers monotonically this same range. Since the endpoints of the interval are identified, one must glue together the two minima of the double-well potential keeping the finite barrier in their middle. The resulting potential is non-analytic at the identification point $0=q=1$, but it remains twice-continuously differentiable there.

The above action has the same gross features as that in [8], namely a harmonic potential around $q=0$ and a barrier height of order $\sim 1 / g^{2}$ at weak coupling. Since $q$ is a compact variable, the energy spectrum is discrete with no bands. Furthermore, in contrast to the non-compact toy model of section 2 here we cannot decree that the shift symmetry is global rather than gauged. Doing this violates locality, because gauge transformations in the topologically trivial $(n=0)$ sector can look 'large' in two widely-separated regions.

One way out is to associate to instanton transitions a shift of some bosonic zero mode ${ }^{4}$ thereby turning configuration space into a helix. Interestingly, this can be achieved without violation of locality by coupling the original Yang-Mills to a topological theory, as proposed by Seiberg [15]. The extra term in the Lagrangian is

$$
\Delta \mathcal{L}_{\mathrm{YM}}=a\left[d \mathcal{C}(A)-\xi^{-1} d B\right],
$$

where $a$ is a Lagrange multiplier field, $B:=\frac{1}{3 !} B_{\mu \nu \rho} d x^{\mu} \wedge d x^{\nu} \wedge d x^{\rho}$ is a 3 -form gauge field, $d$ is the exterior derivative, and $\xi$ is a free parameter - the pitch of the helix.

The quantum-mechanical model on $\mathrm{S}^{3}$ has now two new variables, $a$ and the holonomy of the 3 -form field, $b:=\int_{S^{3}} B$. Their action is

$$
\Delta S=\int d t a\left[\dot{n}_{\mathrm{CS}}-\xi^{-1} \dot{b}\right]
$$

\footnotetext{
${ }^{3}$ Such a reduction was performed in the context of mini-superspace cosmology in ref. [16].

${ }^{4}$ Fermionic zero modes will not do the job, since they at best double the effective period of $n_{\mathrm{CS}}$.
} 
The equation of motion for $b$ forces $a$ to be constant, while the equation of $a$ implies $\xi \dot{n}_{\mathrm{CS}}=\dot{b}$. As expected, this modification did not add any dynamical degrees of freedom to the theory. However, instanton transitions are now accompanied by a shift $\Delta b=\xi \Delta n_{\mathrm{CS}}$, which converts the $n_{\mathrm{CS}}$ circle to a helix.

We may consider $b$ to be a periodic variable, $b \sim b+1$, if we decree that the theory only admits membranes whose charge is an integral multiple of $2 \pi$. By analogy with conventional gauge theories, this means that the admissible volume operators (counterparts of Wilsonloop operators) are labeled by an integer charge $m$,

$$
\mathcal{W}_{m}:=e^{2 \pi i m \int_{S^{3}} B}
$$

The spectrum of the model with action $S+\Delta S$, eqs. (3.3) and (3.5), depends then on the parameter $\xi$. If $\xi=1$ it is the same as for pure Yang-Mills theory. If $\xi$ is irrational the helix is non-compact and the spectrum has continuous bands. Finally, if $\xi=1 / N$ the helix closes after $N$ turns and one should keep $N$ eigenstates in every band.

Let us pick $\xi=1 / N$. The energy eigenvalues in the notation of section 2 are $E_{\theta+2 \pi k / N, \lambda}(0)$ where $\lambda$ is the label of the band and $k=0,1, \cdots, N-1$. Thus $N$ different $\theta$ sectors coexist and the partition function of the theory can be written as

$$
Z=\sum_{k=1}^{N} Z_{\mathrm{YM}}\left(\theta+\frac{2 \pi k}{N}\right),
$$

where $Z_{\mathrm{YM}}(\theta)$ is the partition function of the original Yang-Mills at angle $\theta$. This agrees with the results of Seiberg [15]. Note that local operators do not mix the different $\theta$ sectors - these are superselection sectors in the usual sense. They are however mixed by the operators $\mathcal{W}_{m}$ which shift $\theta$ by $\Delta \theta=2 \pi m / N$, as the reader can verify.

The $\mathcal{W}_{m}$ are examples of boundary or interface operators (also called Janus interfaces when they carry no degrees of freedom, as in this case). They have been studied extensively in $\mathcal{N}=4$ super Yang-Mills where they preserve space-time supersymmetry if combined with an appropriate discontinuity of the coupling $g[22,23]$. More generally, these interfaces can be conformal but not topological as should be clear from the fact that they modify operator dimensions (for a recent discussion of the $\theta$-dependence of scaling dimensions in $\mathcal{N}=4$ super Yang-Mills see [24]). Using radial quantization, the conformal interface operators can be defined on 3 -spheres in $\mathbb{R}^{4}$ and expanded for small 3 -spheres in terms of local operators, the leading of which is the topological density $\operatorname{tr}(F \wedge F)$.

After all the dust has settled the theory looks deceivingly simple. It is a superposition of $N$ distinct gauge theories with different values of the $\theta$-angle, related by interface operators. There is however a non-trivial fact: this coexistence was achieved by a local modification of the field theory action. ${ }^{5}$ The construction is reminiscent of the non-dynamical 3-form introduced by Brown and Teitelboim to describe a multi-valued cosmological constant [26, 27].

\footnotetext{
${ }^{5}$ The same trick can be used to force the coexistence of $2 \mathrm{D}$ sigma models with a Kähler modulus $\tau$ whose real part takes several different values. In free-field models the expansion of interface operators in terms of local operators can be computed to all orders from the exact conformal boundary states, see e.g. [25]. Interestingly, since the dimension of the identity operator vanishes for all values of $\tau$, the 'bandwidth' is zero for the lowest band but nor for those with non-trivial winding.
} 


\section{Dynamical axion}

The 3-form model of the previous section may look contrived, and one can worry whether it arises from a fundamental theory like string theory. It is therefore interesting to see that a similar band structure emerges from a more conventional theory, Yang-Mills coupled to a dynamical axion field. The extra terms in the reduced action are

$$
\Delta S=\int d t\left[\frac{f_{a}^{2}}{2} \dot{a}^{2}-a \dot{n}_{\mathrm{CS}}\right]
$$

where $f_{a}$ is the axion decay constant which we will assume large. We also assume that $a$ is periodic (this is automatic in string-theory embeddings, and maybe more generally [28]) so the target space of our quantum mechanical model is the two-dimensional torus $\left(a, n_{\mathrm{CS}}\right) \sim$ $\left(a, n_{\mathrm{CS}}+1\right) \sim\left(a+2 \pi, n_{\mathrm{CS}}\right)$. Note that $2 \pi$ is the minimal period compatible with a welldefined path-integral measure, but in general the period of $a$ can be $2 \pi k$ with $k \in \mathbb{Z}$. ${ }^{6}$ This freedom will not be important to us, so we set $k=1$.

The action $S+\Delta S$, eqs. (3.3) and (4.1), describes a non-relativistic particle moving on a 2-dimensional torus in the background of a magnetic field and a non-trivial potential. The torus is parametrized by $\left(a / 2 \pi, n_{\mathrm{CS}}\right)$ which we will denote for short $(x, y)$. The background field $A=-2 \pi x d y$ corresponds to one unit of magnetic flux through the torus. Since the quantization of the system does not depend on the choice of gauge for the magnetic field, we may switch to $A=2 \pi y d x$ which amounts to integrating by parts the $\int a \dot{n}_{\mathrm{CS}}$ term of the action. The Hamiltonian is the sum of two terms,

$$
\mathcal{H}=\frac{1}{2 f_{a}^{2}}\left[p_{a}-n_{\mathrm{CS}}\right]^{2}+\mathcal{H}_{0}(\theta)
$$

where $p_{a}$ is the momentum conjugate to $a$, and $\mathcal{H}_{0}(\theta)$ is the Hamiltonian of the pure Yang-Mills theory derived from the action (3.3).

Let us analyze the energy spectrum in steps. We will first ignore the periodicity of $n_{\mathrm{CS}}$ and of the related variable $q$, allowing them to take values on the real line. Recall that $q$ was a redefinition of the Chern-Simons number between successive integers, whose only merit was to simplify the kinetic and potential energy in our toy-action $S$. Since there is nothing sacred about $S$, the fine distinction between $q$ and $n_{\text {CS }}$ plays no special role. The periodicity of $n_{\mathrm{CS}}$ and $q$ will be imposed in the end.

Next, we note that $\mathcal{H}$ commutes with the axion momentum $p_{a}$ which is furthermore quantized, so we may set $p_{a}=n_{a} \in \mathbb{Z}$. The problem now reduces to that of a particle (with canonical kinetic energy and mass $\sim 1 / g^{2}$ ) moving in the effective 1D potential

$$
V\left(q, n_{a}\right)=\frac{1}{2 f_{a}^{2}}\left[n_{a}-n_{\mathrm{CS}}(q)\right]^{2}+V_{0}(q) .
$$

Here $V_{0}$ is the potential of the pure Yang-Mills theory which has been periodically extended from the interval $[0,1]$ to the entire real line.

\footnotetext{
${ }^{6}$ To see why, consider a gauge group $[\mathrm{SU}(2)]^{k}$ broken spontaneously to its diagonal subgroup. The $a \dot{n}_{\mathrm{CS}}$ term in $\Delta S$ is multiplied by $k$, so if normalized as in (4.1) the axion has $k$ times the original period.
} 
We have assumed that the axion scale is much larger than the other scales of the problem, the inverse sphere radius $\left(f_{a} \gg 1\right)$ and the sphaleron's mass $\left(f_{a} \gg 1 / g^{2}\right)$. The spectrum is then, to a good approximation, determined by the periodic potential $V_{0}$ which leads to the characteristic band structure of a lattice model. The only effect of the axion term in (4.3) is to put the particle inside a large box, centered around the Chern-Simons number $n_{\mathrm{CS}}=n_{a}$ and of approximate size $f_{a}$. The box discretizes the quasi-momenta, but since their spacing $\sim 1 / f_{a}$ is small they cover the energy bands rather densely.

We can finally restore the winding transformations to their status of gauge, not global symmetries. In pure Yang-Mills this identified periodically the Chern-Simons number $n_{\mathrm{CS}}$. But (4.3) is only invariant under the combined shifts $\left(n_{\mathrm{CS}}, n_{a}\right) \rightarrow\left(n_{\mathrm{CS}}+n, n_{a}+n\right)$, so this must be the symmetry that we need to gauge. This is familiar from the Landau problem on the square torus $(x, y) \sim(x+1, y) \sim(x, y+1)$. The free-particle Hamiltonian in the $A_{x}=B y$ gauge is $\mathcal{H}=\frac{1}{2}\left(p_{x}-B y\right)^{2}+\frac{1}{2} p_{y}^{2}$. It commutes with the torus translations

$$
U_{x}=e^{i p_{x}} \quad \text { and } \quad U_{y}=e^{i p_{y}} e^{-i B x},
$$

which furthermore commute with each other if $B$ obeys the Dirac quantization $B / 2 \pi=k \in$ $\mathbb{Z}$. The extra factor in $U_{y}$ is the gauge transformation that patches together the local charts at $y$ and $y+1$. The torus identifications impose on wavefunctions the conditions $U_{x} \psi=$ $U_{y} \psi=\psi$, and these are indeed obeyed by the $k$ independent states at each Landau level,

$$
\psi_{\lambda, n_{x}}(x, y)=\sum_{n=-\infty}^{\infty} e^{2 \pi i\left(n_{x}+k n\right) x} \psi_{\lambda}\left(y-n-\frac{n_{x}}{k}\right),
$$

where $\psi_{\lambda}(y)$ are the harmonic-oscillator wavefunctions at level $\lambda$, and $p_{x} / 2 \pi=n_{x} \in$ $\mathbb{Z}(\bmod k)$.

Coming back to our problem we conclude that the large gauge transformations identify periodically $\left(n_{\mathrm{CS}}, n_{a}\right) \sim\left(n_{\mathrm{CS}}+1, n_{a}+1\right)$, thus converting again the configuration space into a helix. The second helix coordinate is the axion momentum $n_{a}$, which is only an approximate zero mode lifted by the small quadratic term in the potential (4.3). In the limit $f_{a} \rightarrow \infty$ the zero mode becomes exact and the splitting of the quasi-momenta vanishes.

That the axion theory gives a similar spectrum as the topological model of the previous section is, actually, no coincidence. We could have argued for this directly by adding to the Lagrangian (3.4) a small mass term for the 3-form field, $\mathcal{L}_{\text {mass }}=\left(\xi f_{a}\right)^{-2} B_{\mu \nu \rho} B^{\mu \nu \rho} / 12$. Integrating out the auxiliary field $B$ gives, after integration by parts, $B_{\mu \nu \rho}=\xi f_{a}^{2} \epsilon_{\mu \nu \rho \sigma} \partial^{\sigma} a$ thus reproducing precisely the dynamical axion model. Since the mass term breaks the periodicity of $B$, interface operators of any charge are now allowed

$$
\mathcal{W}_{\gamma}=e^{i \gamma \int_{S^{3}} * d a} \quad \forall \gamma \in \mathbb{R} .
$$

It is worth stressing that an approximate band structure would also arise from an axion potential with a large number of stable minima between 0 and $2 \pi$, but such a model would be really contrived. The simple axion model considered here is the one emerging naturally from string theory, with $f_{a}$ of order the Planck scale. 
We should here pause to assess the validity of our approximations. Reducing the gauge theory on $\mathrm{S}^{3}$ to a quantum-mechanical model for $n_{\mathrm{CS}}(t)$ has, a priori, zero range of validity. Indeed, the perturbative excitations of this latter model have energy $\sim 1$ (the inverse sphere radius), which is also the energy of the truncated Kaluza-Klein modes. The only thing that this model accurately describes is the $\theta$-dependence of the vacuum energy, or more precisely its exponential sensitivity $e^{-S_{0}}$. This is, however, sufficient for our purpose here, which was to explain how band structure may emerge in Yang-Mills. Note that level-splittings in the lowest band are of order $f_{a}^{-1} e^{-S_{0}} \sim N^{-1} e^{-S_{0}}$, much smaller than the infrared cutoff which is also the gap between bands.

\section{Concluding remarks}

The present investigation was prompted by ref. [8] which challenged the earlier consensus that $B+L$-violating electroweak processes should be invisible at LHC. $^{7}$ The standard wisdom is that the collision energy, initially carried by two hard quanta, has no chance of being streamlined to a coherent excitation of one (or a few) degrees of freedom in a weakly-coupled theory like the electroweak Standard Model. Thus all processes involving solitons as intermediate states should be exponentially suppressed, independently of any other details of the theory at hand.

This 'wisdom' is supported by a lot of theoretical evidence, and also experimental [30, 31]. In the context of the simple quantum mechanics of the Chern-Simons number analyzed here, the suppression would not come from the tunneling rate of an excited state, but rather from the unlikely probability of reaching this state when the energy is initially stored in a 'hard quantum' that can be modelled by a highly-energetic source linear in $q$ [12-14]. Still, in the absence of a definitive calculation and since the LHC Run2 operates in the sphaleron range, it is important to scrutinize all 'heretic' proposals.

We focussed here on one of the issues raised in ref. [8], namely whether the electroweak theory exhibits band structure similar to that of an electron in a solid. We have explained why this is not the case, although it would be conceivable if Yang-Mills theory were coupled to an axion field with high Peccei-Quinn scale and if space could be treated as being compact. It is amusing to note that even this hypothetical band structure would be absent in the electroweak theory for a different reason: because $\theta$ can be rotated away by a chiral $\mathrm{U}(1)_{B}$ and/or $\mathrm{U}(1)_{L}$ rotation. In contrast to what happens for QCD such chiral rotations do not transfer the electroweak $\theta$ angle to the Yukawa couplings of quarks and leptons, so $\theta$ is a relevant parameter only if both $B$ and $L$ are explicitly broken [33].

\section{Acknowledgments}

We thank Ilka Brunner, Cesar Gomez, Slava Mukhanov, Giuseppe Policastro and Jan Troost for discussions. T.T. thanks the Philippe Meyer Institute of the École Normale for support during a visit that initiated this project. C.B. thanks the LMU physics department

\footnotetext{
${ }^{7} \mathrm{~A}$ characteristic signature would be the large number of electroweak bosons produced in the decay of the sphaleron [29-31]. This is similar to the decay of hypothetical black holes in low-scale quantum gravity models for which the ATLAS collaboration has published exclusion plots in [32].
} 
for hospitality during completion of this work. The work of T.T. is partially supported by the European Union Seventh Framework Program (FP7-REGPOT-2012-2013-1) under grant agreement No. 316165 .

\section{A An action for the Chern-Simons number}

A prototypical action for the Chern-Simons number $n_{\mathrm{CS}}$ can be found by putting YangMills theory on $\mathrm{S}^{3} \times \mathbb{R}_{\tau}$ where $\mathrm{S}^{3}$ is the unit-radius round 3-sphere and $\tau$ is imaginary time. This spacetime is related to Euclidean $\mathbb{R}^{4}$ by a Weyl transformation,

$$
d s^{2}\left[\mathrm{~S}^{3} \times \mathbb{R}\right]=d \tau^{2}+d \Omega_{3}^{2}=\frac{1}{r^{2}}\left(d r^{2}+r^{2} d \Omega_{3}^{2}\right)=\frac{1}{r^{2}} d s^{2}\left[\mathbb{R}^{4}\right]
$$

with $\tau=\log r$, and $d \Omega_{3}^{2}$ the metric on the round 3-sphere. Since the self-dual Yang-Mills equations are Weyl-invariant, ${ }^{8}$ all solutions in $\mathbb{R}^{4}$ also solve the equations in $S^{3} \times \mathbb{R}$. This is in particular true for the celebrated Belavin et al. [20] instanton

$$
A_{\mu}=-\frac{\bar{\sigma}_{\mu \nu}\left(x^{\nu}-a^{\nu}\right)}{(x-a)^{2}+\rho^{2}} \quad \Longrightarrow \quad F_{\mu \nu}=\frac{2 \bar{\sigma}_{\mu \nu} \rho^{2}}{\left[(x-a)^{2}+\rho^{2}\right]^{2}}
$$

where $\bar{\sigma}_{\mu \nu}=-i \eta_{\mu \nu}^{a} \sigma^{a}, \eta$ is the 't Hooft tensor and $\sigma^{a}$ the Pauli matrices. We are following here the conventions of reference [21]. The instanton center, $a^{\nu}$, and the instanton scale, $\rho$, are collective coordinates of the solution.

To express this solution in terms of $\tau$ and coordinates $\phi_{m}$ on the 3 -sphere we use the relation $x^{\mu}=|x| \hat{n}^{\mu}=e^{\tau} \hat{n}^{\mu}\left(\phi_{m}\right)$ where $\hat{n}_{\mu} \hat{n}^{\mu}=1$. The instanton centered at $a^{\nu}=0$, which preserves an $\mathrm{O}(4)$ symmetry, is automatically in the $A_{\tau}=0$ gauge thanks to the antisymmetry of $\bar{\sigma}_{\mu \nu}$. The vector potential, and the electric and magnetic fields read

$$
A_{m}=\frac{\bar{\sigma}_{\mu \nu} \hat{n}^{\mu} \partial_{m} \hat{n}^{\nu}}{1+e^{2\left(\tau_{0}-\tau\right)}}, \quad F_{\tau m}=\frac{\bar{\sigma}_{\mu \nu} \hat{n}^{\mu} \partial_{m} \hat{n}^{\nu}}{2 \cosh ^{2}\left(\tau-\tau_{0}\right)}, \quad F_{l k}=\frac{\bar{\sigma}_{\mu \nu} \partial_{l} \hat{n}^{\mu} \partial_{k} \hat{n}^{\nu}}{2 \cosh ^{2}\left(\tau-\tau_{0}\right)} .
$$

We have here defined $\rho \equiv e^{\tau_{0}}$ to make clear that the instanton scale in $\mathbb{R}^{4}$ becomes the time collective coordinate in the new coordinate system. ${ }^{9}$ The gauge field vanishes at $\tau \rightarrow-\infty$ and is pure gauge at $\tau \rightarrow+\infty$, so its field strength vanishes at both ends as expected since it describes vacuum-to-vacuum tunneling.

From now on we set $\tau_{0}=0$ and think of (A.3) as a path in configuration space parametrized by $\tau$. The potential energy along the path is the energy stored in the magnetic fields,

$$
V(\tau)=-\frac{1}{2 g^{2}} \int_{\mathrm{S}^{3}} \operatorname{tr}\left(F_{l k} F^{l k}\right)=\frac{3 \pi^{2}}{g^{2}} \frac{1}{\cosh ^{4} \tau}, \quad V_{\max }=\frac{3 \pi^{2}}{g^{2}} .
$$

\footnotetext{
${ }^{8}$ The self-duality equations are $F_{\mu \nu}=\frac{1}{2} \epsilon_{\mu \nu}{ }^{\rho \sigma} F_{\rho \sigma}$, where the Levi-Civita tensor obeys $\sqrt{|g|} \epsilon^{\mu \nu \rho \sigma} \equiv \hat{\epsilon}^{\mu \nu \rho \sigma}$ with $\hat{\epsilon}$ the totally antisymmetric symbol normalized to \pm 1 . Clearly $\epsilon_{\mu \nu}{ }^{\rho \sigma}$ is Weyl invariant.

${ }^{9}$ This is familar from holographic dualities, where one identifies the instanton moduli with the coordinates of a D-instanton in $\mathrm{AdS}_{5}$. The moduli $a^{\nu}$ and $\rho$ are Poincaré coordinates for $\mathrm{AdS}_{5}$, while $\tau_{0}$ is the time in global coordinates. Setting $a^{\nu}=0$ amounts to putting the D-instanton in the center of global $\mathrm{AdS}_{5}$.
} 
The normalization can be quickly fixed by noting that the integral of $V$ is half the instanton action, $\int d \tau V(\tau)=\frac{4 \pi^{2}}{g^{2}}$. The energy barrier is highest at $\tau=0$, the analog of the sphaleron of the Standard Model. Note that the role of the W-boson mass is played by the radius of the 3 -sphere, which introduces a cutoff on the instanton size. In holographic language the barrier height attains its (non-zero) minimum when the D-instanton sits at the center of $\mathrm{AdS}_{5}$.

One may generalize the gauge field (A.3) to a variational ansatz (in temporal gauge) that depends on a single arbitrary function $y(\tau)$,

$$
A_{\tau}=0, \quad A_{m}=\frac{\bar{\sigma}_{\mu \nu} \hat{n}^{\mu} \partial_{m} \hat{n}^{\nu}}{1+e^{-2 y(\tau)}} .
$$

It can be checked that this is the most general $\mathrm{SO}(4)$-invariant configuration of the gauge fields, so this is a symmetry truncation of configuration space. There is a single surviving degree of freedom, which we can take to be the Chern-Simons number $n_{\mathrm{CS}}$. The magnetic fields for the above configuration are the same as in (A.3) with $\tau$ replaced by $y(\tau)$, while the electric fields are also multiplied by $\dot{y}$. Notice that this is not a reparametrization since we keep the same metric $g_{\tau \tau}=1$. Computing the energy of the electric and magnetic fields gives the effective Lagrangian (in Euclidean time) ${ }^{10}$

$$
\mathcal{L}(q, \dot{q})=\frac{3 \pi^{2}}{g^{2}} \frac{1}{\cosh ^{4} y}\left(\dot{y}^{2}+1\right) .
$$

One can check that $\dot{y}=1$ solves the variational equation for this action, so the instanton is a solution as it should be. The change of variable $z=\tanh y$ transforms the problem to the standard double-well quantum mechanics,

$$
\mathcal{L}(q, \dot{q})=\frac{3 \pi^{2}}{g^{2}}\left[\dot{z}^{2}+\left(z^{2}-1\right)^{2}\right] .
$$

Finally we can relate the variable $z$ to the Chern-Simons number of the field,

$$
n_{\mathrm{CS}}(q)=-\frac{1}{8 \pi^{2}} \int_{\mathrm{S}^{3}} \epsilon^{l k m} \operatorname{tr}\left(A_{l} \partial_{k} A_{m}+\frac{2}{3} A_{l} A_{k} A_{m}\right)
$$

Using the fact that space is compact and that $n_{\mathrm{CS}}(-\infty)=0$, one can rewrite the above integral as a 4-dimensional integral of $\operatorname{tr}\left(F_{\mu \nu}{ }^{*} F^{\mu \nu}\right)$. This does not involve the metric, so the result is form-invariant whether expressed as function of $\tau$ or $y$. Performing the integral gives

$$
n_{\mathrm{CS}}=\frac{1}{2}+\frac{\sinh (3 y)+3 \sinh y}{8 \cosh ^{3} y}=\frac{1}{2}+\frac{z}{4}\left(3-z^{2}\right) .
$$

In principle, one can invert this relation to find an effective quantum mechanics

$$
\mathcal{L}=\frac{1}{2} M\left(n_{\mathrm{CS}}\right) \dot{n}_{\mathrm{CS}}^{2}-V\left(n_{\mathrm{CS}}\right)
$$

with functions $M$ and $V$ that can be computed.

\footnotetext{
${ }^{10}$ The general Lagrangian that admits $\dot{y}=1$ as a solution is $\mathcal{L}=f(y)\left(\dot{y}^{2}+1\right)$. Our calculation of the energy stored in the magnetic fields fixes the arbitrary function $f(y)$.
} 
Note that the range of $z$ is $[-1,1]$, and the corresponding range of $n_{\mathrm{CS}}$ is $[0,1]$, so the Lagrangians (A.7) and (A.10) should be restricted to these finite intervals. A last change of variable to $q=(z+1) / 2$, and Wick rotation to real time, gives finally the expressions (3.2) and (3.3) of section 3. The range of $q$ is the same as the range of $n_{\mathrm{CS}}$, so $q$ can be considered as a convenient redefinition of the Chern-Simons number. Since the only features of the action that are relevant to our discussion are (1) its periodicity, and (2) the fact that the potential barrier traversed by winding histories is finite, the fine distinction between $n_{\mathrm{CS}}$ and $q$ is not important.

Open Access. This article is distributed under the terms of the Creative Commons Attribution License (CC-BY 4.0), which permits any use, distribution and reproduction in any medium, provided the original author(s) and source are credited.

\section{References}

[1] G. 't Hooft, Symmetry breaking through Bell-Jackiw anomalies, Phys. Rev. Lett. 37 (1976) 8 [INSPIRE].

[2] F.L. Bezrukov, D. Levkov, C. Rebbi, V.A. Rubakov and P. Tinyakov, Semiclassical study of baryon and lepton number violation in high-energy electroweak collisions, Phys. Rev. D 68 (2003) 036005 [hep-ph/0304180] [INSPIRE].

[3] F.L. Bezrukov, D. Levkov, C. Rebbi, V.A. Rubakov and P. Tinyakov, Suppression of baryon number violation in electroweak collisions: Numerical results, Phys. Lett. B 574 (2003) 75 [hep-ph/0305300] [INSPIRE].

[4] A. Ringwald, An upper bound on the total cross-section for electroweak baryon number violation, JHEP 10 (2003) 008 [hep-ph/0307034] [INSPIRE].

[5] A. Ringwald, Electroweak instantons/sphalerons at VLHC?, Phys. Lett. B 555 (2003) 227 [hep-ph/0212099] [INSPIRE].

[6] M.P. Mattis, The riddle of high-energy baryon number violation, Phys. Rept. 214 (1992) 159 [INSPIRE].

[7] R. Guida, K. Konishi and N. Magnoli, Fermions, anomaly and unitarity in high-energy electroweak interactions, Int. J. Mod. Phys. A 9 (1994) 795 [hep-ph/9311219] [InSPIRE].

[8] S.H.H. Tye and S.S.C. Wong, Bloch wave function for the periodic sphaleron potential and unsuppressed baryon and lepton number violating processes, Phys. Rev. D 92 (2015) 045005 [arXiv: 1505.03690] [inSPIRE].

[9] S.H.H. Tye and S.S.C. Wong, The Chern-Simons number as a dynamical variable, arXiv: 1601.00418 [INSPIRE].

[10] A. Ringwald, High-energy breakdown of perturbation theory in the electroweak instanton sector, Nucl. Phys. B 330 (1990) 1 [INSPIRE].

[11] O. Espinosa, High-energy behavior of baryon and lepton number violating scattering amplitudes and breakdown of unitarity in the standard model, Nucl. Phys. B 343 (1990) 310 [INSPIRE].

[12] C. Bachas, G. Lazarides, Q. Shafi and G. Tiktopoulos, Quantum mechanical tunneling at high-energy, Phys. Lett. B 268 (1991) 401 [Erratum ibid. B 271 (1991) 468] [INSPIRE].

[13] C. Bachas, On the breakdown of perturbation theory, Theor. Math. Phys. 95 (1993) 491 [Teor. Mat. Fiz. 95 (1993) 187] [hep-th/9212033] [INSPIRE]. 
[14] C. Bachas, A proof of exponential suppression of high-energy transitions in the anharmonic oscillator, Nucl. Phys. B 377 (1992) 622 [INSPIRE].

[15] N. Seiberg, Modifying the sum over topological sectors and constraints on supergravity, JHEP 07 (2010) 070 [arXiv: 1005.0002] [InSPIRE].

[16] G.W. Gibbons and A.R. Steif, Yang-Mills cosmologies and collapsing gravitational sphalerons, Phys. Lett. B 320 (1994) 245 [hep-th/9311098] [InSPIRE].

[17] V. Rubakov, Classical theory of gauge fields, Princeton University Press, Princeton U.S.A. (2002).

[18] E. Witten, Theta dependence in the large-N limit of four-dimensional gauge theories, Phys. Rev. Lett. 81 (1998) 2862 [hep-th/9807109] [INSPIRE].

[19] F.R. Klinkhamer and N.S. Manton, A saddle point solution in the Weinberg-Salam theory, Phys. Rev. D 30 (1984) 2212 [INSPIRE].

[20] A.A. Belavin, A.M. Polyakov, A.S. Schwartz and Yu.S. Tyupkin, Pseudoparticle solutions of the Yang-Mills equations, Phys. Lett. B 59 (1975) 85 [InSPIRE].

[21] S. Vandoren and P. van Nieuwenhuizen, Lectures on instantons, arXiv:0802.1862 [INSPIRE].

[22] E. D'Hoker, J. Estes and M. Gutperle, Exact half-BPS type IIB interface solutions. I. Local solution and supersymmetric Janus, JHEP 06 (2007) 021 [arXiv:0705.0022] [INSPIRE].

[23] D. Gaiotto and E. Witten, Janus configurations, Chern-Simons couplings, and the theta-angle in $N=4$ super Yang-Mills theory, JHEP 06 (2010) 097 [arXiv:0804.2907] [INSPIRE].

[24] C. Beem, L. Rastelli, A. Sen and B.C. van Rees, Resummation and S-duality in $N=4 S Y M$, JHEP 04 (2014) 122 [arXiv: 1306. 3228] [INSPIRE].

[25] C. Bachas, I. Brunner and D. Roggenkamp, A worldsheet extension of $O(d, d: Z)$, JHEP 10 (2012) 039 [arXiv: 1205.4647] [INSPIRE].

[26] J.D. Brown and C. Teitelboim, Dynamical neutralization of the cosmological constant, Phys. Lett. B 195 (1987) 177 [INSPIRE].

[27] J.D. Brown and C. Teitelboim, Neutralization of the cosmological constant by membrane creation, Nucl. Phys. B 297 (1988) 787 [INSPIRE].

[28] T. Banks and N. Seiberg, Symmetries and strings in field theory and gravity, Phys. Rev. D 83 (2011) 084019 [arXiv: 1011.5120] [INSPIRE].

[29] M.J. Gibbs, A. Ringwald, B.R. Webber and J.T. Zadrozny, Monte Carlo simulation of baryon and lepton number violating processes at high-energies, Z. Phys. C 66 (1995) 285 [hep-ph/9406266] [INSPIRE].

[30] J. Ellis and K. Sakurai, Search for sphalerons in proton-proton collisions, JHEP 04 (2016) 086 [arXiv : 1601.03654] [INSPIRE].

[31] J. Ellis, K. Sakurai and M. Spannowsky, Search for sphalerons: IceCube vs. LHC, JHEP 05 (2016) 085 [arXiv: 1603.06573] [INSPIRE].

[32] ATLAS collaboration, Search for strong gravity in multijet final states produced in $p p$ collisions at $\sqrt{s}=13$ TeV using the ATLAS detector at the LHC, JHEP 03 (2016) 026 [arXiv: 1512.02586] [INSPIRE].

[33] A.A. Anselm and A.A. Johansen, Can electroweak theta term be observable?, Nucl. Phys. B 412 (1994) 553 [hep-ph/9305271] [INSPIRE]. 